\title{
MODROB: THE HARDWARE-SOFTWARE FRAMEWORK FOR Modular Mobile Robots PRototyPing
}

\author{
Victor Andreev, Valerii Kim \& Stanislav Eprikov
}
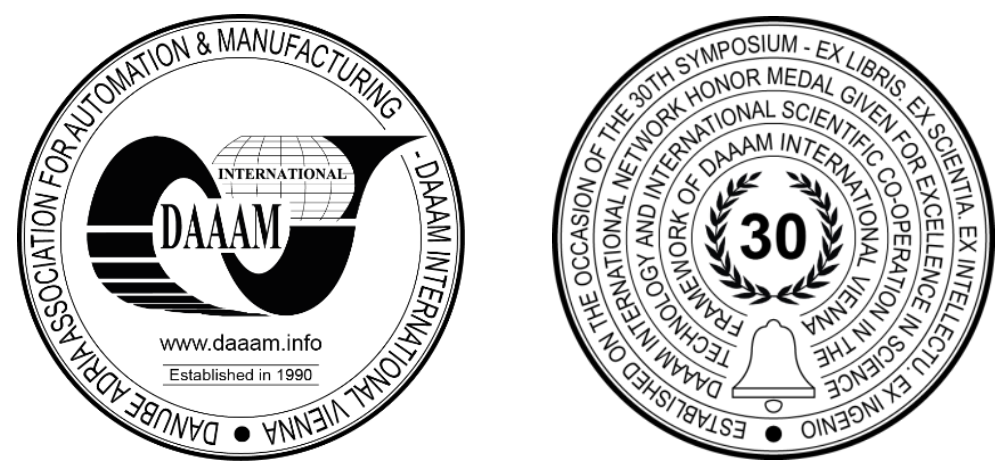

This Publication has to be referred as: Andreev, V[iktor]; Kim, V[alerii] \& Eprikov, S[tanislav] (2020). ModRob: the Hardware-Software Framework for Modular Mobile Robots Prototyping, Proceedings of the 31st DAAAM International Symposium, pp.0391-0402, B. Katalinic (Ed.), Published by DAAAM International, ISBN 978-3-90273429-7, ISSN 1726-9679, Vienna, Austria

DOI: $10.2507 / 31$ st.daaam.proceedings.054

\begin{abstract}
In this paper we consider the hardware-software framework for solving problems associated with the integration of various robotic components into a single robotic system. As the complexity of navigation algorithms for mobile robots is constantly increasing, new approaches to the design of their control systems based on their modular architecture are required. The purpose of this work is to present the modular architecture of reconfigurable mobile robots as a hierarchical structure of control signal handlers. In this architecture a mobile robot is considered as a combination of modules, which in turn consist of simpler units - submodules. Each submodule includes a low-power microcontroller or an single-board computer and is responsible only for basic functions. A full-featured module is formed from a set of such submodules: a transport platform, a robot arm, a leg and so on. The article describes the manufactured prototypes of the submodules, briefly discusses the custom protocol of intermodular interaction of submodules connected by a CAN bus. The results of experiments on testing the protocol for providing real-time mode are presented and their analysis is given. The efficiency of the proposed solution, limitations and a short plan of further actions for the implementation of the project are shown.
\end{abstract}

Keywords: modular robot; mobile robot; distributed systems; communication protocol; CAN.

\section{Introduction}

One of the most common approaches to the design of technical devices and ensuring their versatility is the division of the structure of a technical system into separate functional units - modules. The modular approach is widely known in the automotive, machine tool and computer industries. For example, in computers, almost all major components are standardized, and the interfaces for their communication are well developed. This allows almost any experienced user to assemble a personal computer using compatible module components. Currently, modular robotic systems are actively developing, and they are no longer limited to laboratory prototypes, but are beginning to appear on the market [1], [2].

The modular approach in mobile robotics is aimed at solving a significant problem - ensuring the integration and interoperability of a wide range of hardware and software components of a specific robotic system. Modern robotics professionals face the common problem of "reinventing the wheel" - a significant portion of the time is spent integrating hardware/software interfaces instead of implementing new algorithms. 
Another problem is that the successful performance of tasks by robots in real conditions requires the onboard computer to perform a large number of mathematical calculations with data of different types in real time. The computational load increases even more when it is necessary to process video streams and navigate with the terrain map building. A modular approach to mobile robots design can solve this problem if the calculations performed on one computer are distributed among the computing devices of the modules.

The purpose of the work is to present the hardware and software framework ModRob (ModRob framework) - a hierarchical modular architecture for mobile robots that allows you to accelerate the development of robotic systems through the use of unified components (modules). This project was briefly introduced in the article [3], but in the present article we further describe our new solutions and results. The article is organized as follows: Section 2 provides a brief overview of similar studies, Section 3 discloses in detail the proposed modular architecture and the main types of modules being developed, Section 4 briefly describes the protocol of intermodular communication and hardware of submodules, Section 5 describes the experiments and analyzes the obtained results. At the end of the article, conclusions are given.

\section{Survey}

Researchers working on modular robot architectures strive to achieve basically two goals:

1. Facilitate the work of other scientists and engineers by providing unified robotic components and / or generic programming interfaces. As noted earlier, today there is a wide variety of incompatible hardware and software components of robots that must be combined into a single system.

2. Development of robots for tasks of a non-deterministic nature. The modular approach allows for the creation of reconfigurable robots suitable for missions in unpredictable environments.

The first goal closely intersects with the development of hardware and software frameworks, middlewares and operating systems (OS) for robots (for example, ROS). Frameworks, middlewares and operating systems share the same goal: to provide tools for rapid robot prototyping. An analytical review of such systems has shown that most of them work mainly with robot software, without affecting the hardware component. Detailed reviews of existing frameworks have already been published by other authors, for example [4]. Therefore, we will focus our attention on research that includes not only the software, but also the hardware of modular mobile robots.

The article [5] presents a modular mini-robotic platform AMiRo, which is based on the idea of distributing the computational load between different modules. The platform contains powerful hardware and an advanced open software architecture. The standard robot configuration contains three basic modules: a motion module, a status visualization and wireless communication module and a power module (power supply). The modules have a fairly versatile but complex electrical interface: transmission of five different voltage levels, support for 6 communication standards (the main one is CAN). The software architecture of the modules is quite non-trivial, but it provides wide opportunities for working with third-party applications. However, adding third-party modules to the system may be difficult due to the multi-level software architecture and the small size of the robot.

In [6] a set of modules, mainly modular drives with sequential elastic elements, is described, which makes it possible to speed up the development of modular walking robots. Each drive module controls one brushless DC motor by position, speed, and torque. In addition to the drive modules, there are also specialized ones: sensor modules, a Swedish wheel module, and a head module. All modules are implemented on ARM Cortex series microcontrollers; inter-module information communication is carried out over the network using a family of Ethernet technologies using UDP/TCP protocols. The advantages of the system include distributed computing, easy reconfiguration, and the ability to connect third-party devices. However, the distribution of computing resources is performed only at the lower level of the modular architecture of the robot's control system, which will not solve the real-time problem when the robot's target function becomes more complex.

The CLAWAR international project [7] was launched in 1997 and was coordinated by the University of Portsmouth. The CLAWAR project developed the concept of functional modules, requirements and specifications for them. According to the concept, the object-oriented approach was used for the methodology of designing modular components. The project describes three main types of modules: human-machine interface, actuator modules, and sensor modules. Compared to other similar architectures, Virk G. S. represents a hierarchical modular architecture: the entire system is divided into modules, which in turn are divided into submodules. The concept of CLAWAR is quite promising, but according to information from open sources, this architecture has not yet been implemented in any of the modern robots.

The article [8] presents a modular architecture for Autonomous mobile robots based on the so-called "continuous" modularity of hardware and software. The hardware consists of four levels: the motion level, the sensor level, the actuator level, and the control level. Inter-module communication is based on the CAN bus. The software architecture is implemented on MIRO middleware. The effectiveness of robots was shown at the RoboCup 2003 competition, for which a series of robots was made in less than a year. Despite the presence of distributed computing, the authors do not disclose the details of the communication process between modules, which does not allow us to judge the presence of the reconfigurability property using third-party modules. 
Modular reconfigurable anthropomorphic robots are presented in [9], [10] and [11]. In [9], distributed computing is performed on functional modules: head module, main module, left/right arm, wheel module. The authors developed their own real-time communication interface, Responsive Link, for inter-module interaction. In addition, the communication interface also includes two USB ports and IEEE 1394 ports. Using the proposed architecture, the authors made a reconfigurable anthropomorphic robot. The main drawback of the system is the need to use a special built-in processor (Responsive Processor) that can support the Responsive Link interface, which complicates the integration of third-party components. In [10], a similar approach to designing a humanoid robot is proposed. According to this approach, all modules are fully functional devices, and the modules are able to interact with each other, even if different operating systems are installed on their computers. This property is achieved through a software architecture and a special software connection architecture that defines 5 information model standards. Inter-module communication is based on TCP / IP and RS485. Hild M. and Spranger M. also developed the Myon modular anthropomorphic robot with the DISTAL distributed control architecture [11]. The main features of the robot: autonomous operation of modules and the ability to reconfigure even when the robot is turned on. For inter-module information interaction, the authors developed two communication buses: Spinal Cord and Extended Spinal Cord. The disadvantages of the system include the lack of a software interface between the modules, as well as the presence of distributed computing only at the drive control level.

The concept of a modular service robot DRP I is presented in [12]. DRP I consists of fully functional modules on single-board computers and additional electronic units for management and testing. Each module's software consists of an OS layer, a virtual machine layer, an application programming interface (API), and an application software. The robot's middleware is based on IEEE 1394. Implemented hot plug of robot modules with automatic reconfiguration of navigation algorithms. Despite all the advantages of the presented architecture, there are no modern robotic solutions based on it.

A group of researchers in [13] presented a paradigm for building distributed architectures of mobile robot control systems - ASEBA. ASEBA is a fully centralized system consisting only of microcontrollers that control sensors and actuators. All modules (microcontrollers) are connected by a CAN bus. In ASEBA control is implemented on events at the expense of virtual machines running on microcontrollers. From our point of view, this architecture will not ensure the operation of a robotic system in real time if even not very complex work is performed.

The article [14] presents the R2P system, which is similar to our project. R2P is an open hardware and software framework that allows you to combine various modules in a plug-and-play mode. R2P modules are implemented on ARM Cortex-M3 microcontrollers that interact over the CAN bus using the author's RTCAN protocol. The disadvantage of RTCAN is the fragmentation of all messages, which can lead to collisions of CAN frames, and also reduces the speed of message transmission. Also, from the material given in the article, it is not clear on what principle a particular R2P software node should be deployed on a hardware module, which does not lead to an understanding of the requirements for the computing resources of the modules.

The RoboCAN framework, which has similarities to R2P [15], is also a modular architecture on the CAN bus. RoboCAN, in turn, is part of the RIDE framework. All modules are based on microcontrollers and support plug-andplay mode. It is worth noting that RoboCAN uses its own protocol, which is slightly better than the well-known CANOpen: the bandwidth of the communication channel in RoboCAN is only $25 \%$ less than in CANOPen (when the drives are not working).

The article [16] presents a kit for prototyping modular mobile robots mainly for educational purposes. The kit includes various block modules: control module, power supply, drive modules, data transmission modules, gearboxes, etc. The modules are connected via the RS485 bus or directly to the control module. The kit modules have built-in electronic/electromechanical devices (the module's electronic board is not disconnected from the actuator). This approach makes it easy to assemble mobile robots, but limits the scope of the kit: for example, if the user needs more powerful engines or other sensors.

The ambitious H-ROS project is presented in the article [17]. The goal of the project is to develop a universal hardware and software framework for integration and interoperability of various robot components. H-ROS is based on ROS, but uses the functionality of the latest OS for robots - ROS 2. The physical layer of inter-module communication is based on EtherCAT. Above the physical layer is the Linux kernel with the real-time PREEMPT_RT patch. The disadvantages of the system include the need to use a computationally powerful single-board computer in each module (for deploying Linux and ROS 2), which is redundant for modules that perform low-level control of executive devices. In addition, EtherCAT technology is quite expensive for inter-module information interaction. At the moment, the HROS project has been stopped by developers.

In [18] the concept of a modular robot architecture based on the so-called Operator-Controller Module (OCM) is presented. The novelty of the concept lies in the modular hierarchical structure of the robot and the organization of distributed computing on the local part (the robot itself) and the remote part (cloud computing). The remote part significantly increases the computing power of the system for complex algorithms without increasing the robot's power consumption, but significantly reduces the autonomy of the mobile part and the reliability of the robotic system. The authors developed a prototype of a mobile robot with a distributed control system that includes two raspberry Pi singleboard computers, an Arduino Mega, the STM32 Discovery platform and the ZynqBerry TE0726-03M FPGA. All devices except FPGAs are connected by a CAN bus. Note that this architecture does not unify the robot components (modules) in any way, which may complicate reconfiguration. 
A brief analysis of the presented works showed that not all architectures and frameworks are developed enough to provide a reliable tool for other specialists to quickly prototype robotic solutions. However, as a rule, reconfigurability is provided within the proposed kit of modules, which does not allow you to fully implement the possibility of using third-party modules. In addition, most of the works consider distributed computing only at the low level (drive control), while the implementation of distributed computing of the most complex algorithms for data control and fusion is practically not considered. Also note that some of the presented systems require the use of powerful single-board computers, which partially weakens the positive effect of the modular approach.

\section{Hierarchical modular architecture of reconfigurable mobile robot}

Within the framework of this project, it is proposed to use a pyramidal modular architecture for mobile robots for various purposes [19]. Figure 1 shows a diagram of such a generalized hierarchical structure of the modular mobile robot control system.

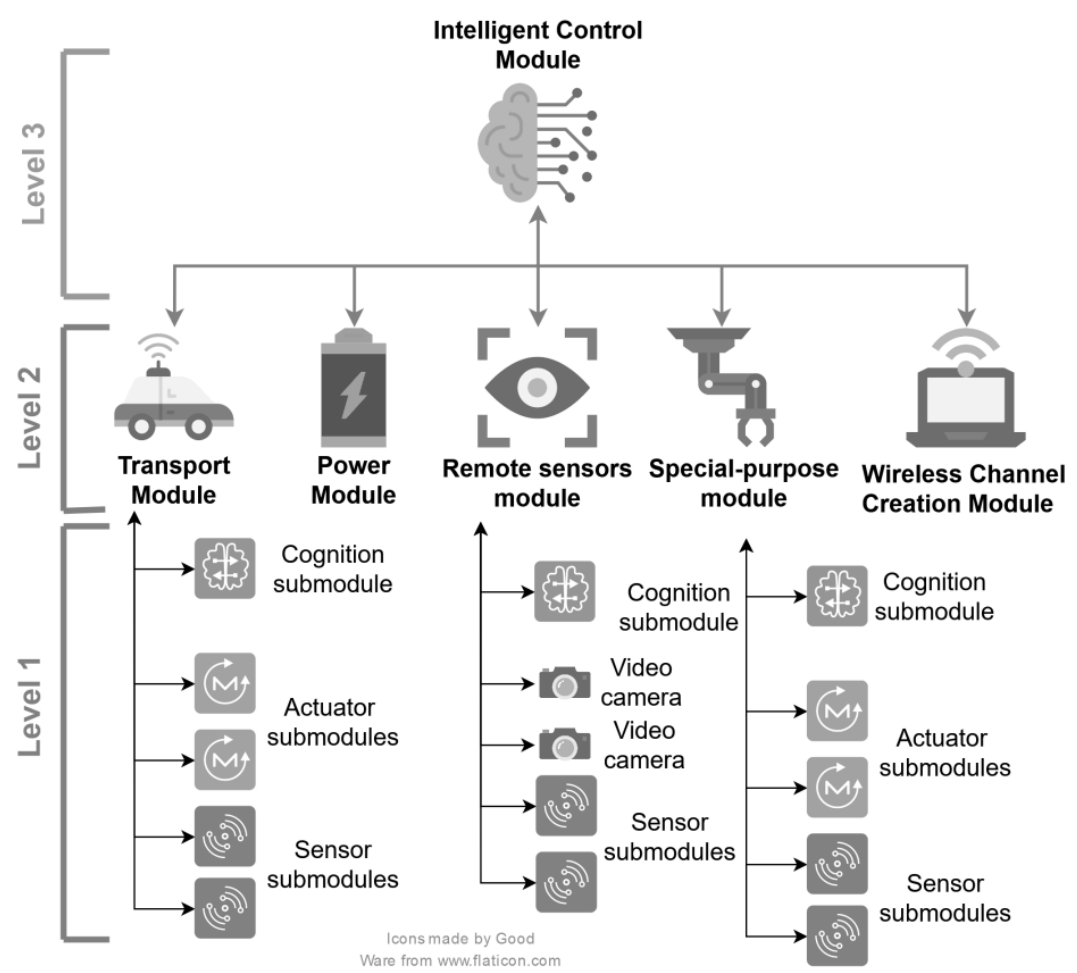

Fig. 1. The generalized hierarchical structure of modular mobile robot control system

At the top of the architecture is an intelligent control module that is responsible for planning and distributing tasks between second-level modules (system-wide control). In this architecture, modules at the third and second levels of the hierarchy are responsible for one enlarged function of the entire robot according to the principle of full functionality [20]: each robot module should be able to perform its goal function in any convenient way, using only its own means to execute commands from an external control system. In general, the control system structure of a typical mobile robot can be divided into the following main functional modules [21]:

1. Intelligent control module - planning and distribution of tasks between the modules of the second level (the system-wide control function).

2. Transport module - robot's motion in an environment (the transport function).

3. Power module - provides power to the robot components (the power function).

4. Remote sensor module - search for obstacles and manipulation objects (the information function).

5. Special-purpose module - the technological function.

6. Wireless channel creation module - communication with the external supervisor (the communication function).

The division of the structure of the mobile robot control system into such blocks is due to the presence of all the functions listed above in almost any mobile robot with elements of intelligent or adaptive control [22], [23], [24]. In a modular robot, the blocks responsible for various functions still need to interact directly or indirectly for the system to function in a consistent way. 
Some second-level modules must perform a large amount of work. Therefore, according to our ModRob concept, each fully functional module should also be built as a modular architecture with submodule nodes. This architecture will allow to distribute the computational load of various functional significance between submodules, especially in cases where the number of drives and sensors may increase or their type may change. Additionally, one can combine modules at both the first and second levels of the hierarchy, respectively. Consider the distributed architecture of the control system of a full-featured Transport module (fig. 2).

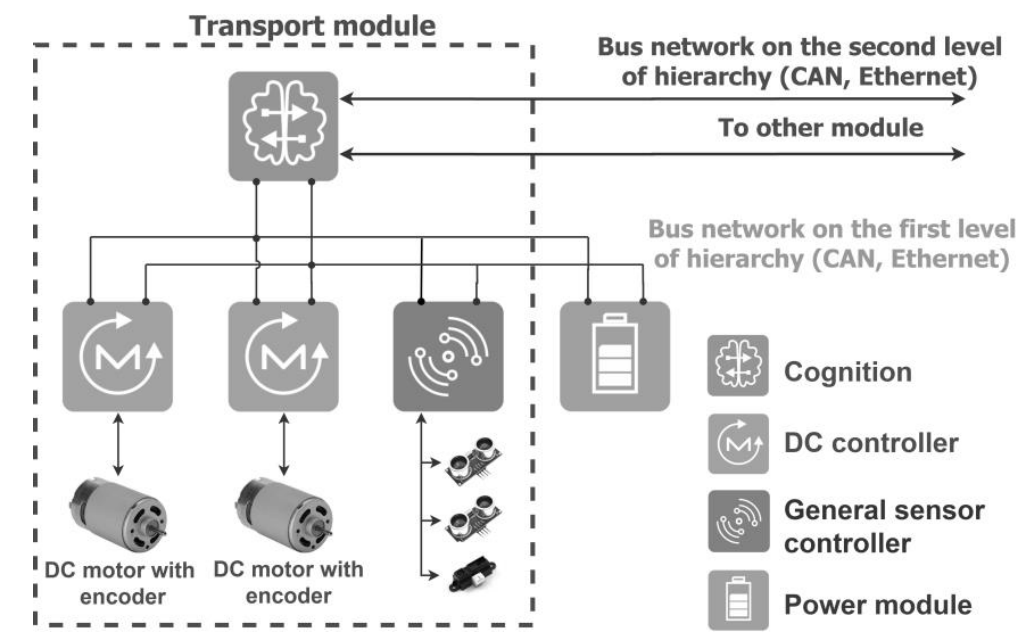

Fig. 2. The distributed architecture of the transport module control system

In this hierarchical structure, the Transport module interacts with other full-featured robot nodes in the network at the second level of the hierarchy (for example, using CAN, Ethernet, or USB communication channels). It is assumed that this interaction can be performed in "soft" real-time mode.

The structure of the Transport module is based on three types of submodules:

1. The Cognition submodule - responsible for the administration of submodules on the bus.

2. The Actuator submodule - control connected electromotor (fig. 2 shows partial case - sub-module for DC control).

3. The General Sensor submodule - allows connecting a variety of distance sensors, encoders, gyroscopes, etc.

The presence of a General Sensor submodule in the Transport module is optional; it can be used in situations where the Sensor module (at the top level of the hierarchy) is not present in the mobile robot and there is a high risk of collision with obstacles. Figure 2 also shows the Power module that provides power to all connected devices. The Power module is not a part of the Transport module and is considered as a specific node of the presented system, since it provides information interaction with modules (the second level of the hierarchy), but provides power to both modules and submodules. Information interaction between submodules should be carried out in real time mode. CAN is currently selected as the primary transport protocol for the first level of the hierarchy. The motivation for this choice will be given below. Consider in detail the design of submodules.

The Cognition submodule is a computing device whose main functions are programmed by the user. For example, the Cognition submodule can be responsible for setting the linear and angular velocities of the transport module according to an algorithm, calculating the robot's position in space, and so on. The Cognition submodule also acts as a "bridge" between the high-level computer and other submodules (Fig. 3). This is necessary for configuring and debugging submodules. The submodule can be connected to a computer via wired (USART) or wireless (Wi-Fi) link.

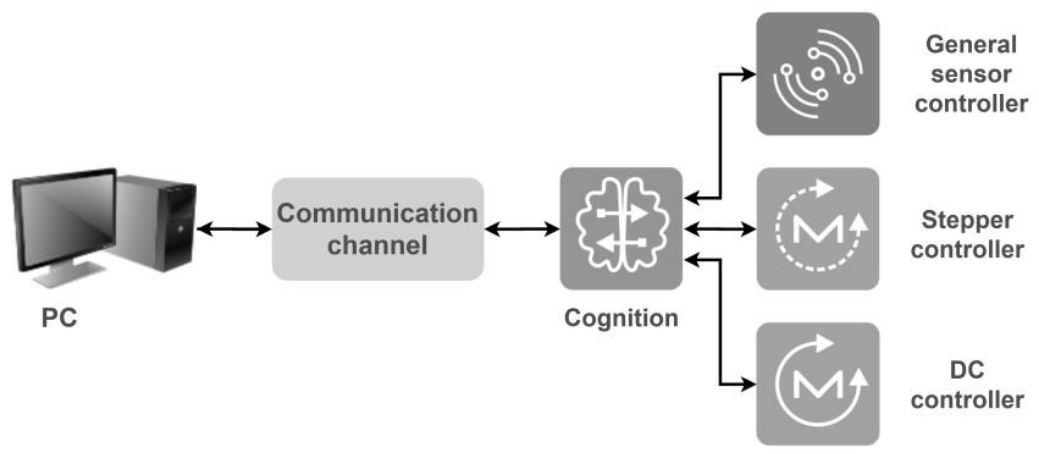

Fig. 3. Connecting a group of submodules to a PC via the Cognition submodule 
One of the main functions of the Cognition submodule is to collect, store, and update the list of submodules connected to the bus. The Cognition submodule must aggregate information about currently active submodules, keep records of nodes publishing on the bus, and regulate subscriptions of some submodules to messages from others.

The Actuator submodule is an electronic device that controls various electric motors: brushed, brushless DC motors, stepper motors, servomotors. Main functions of the submodule: control of the motor shaft by speed, position and/or torque. Depending on the type of regulated electric motor, submodule drives can be of four main types: brushed DC controller, brushless DC controller, stepper motor controller, and servomotor controller.

The General Sensor submodule - an electronic device designed for connecting various sensors, mainly rangefinders, encoders, limit switches, as well as inertial sensors (gyroscopes, accelerometers). The main functions of the submodule: collection and processing of sensory data, data fusion. An important feature of the submodule is the ability to connect different models of sensors of the same type, for example, ultrasonic rangefinders. It is assumed that the submodule should be compatible with the most common standard sensors (Fig. 4).

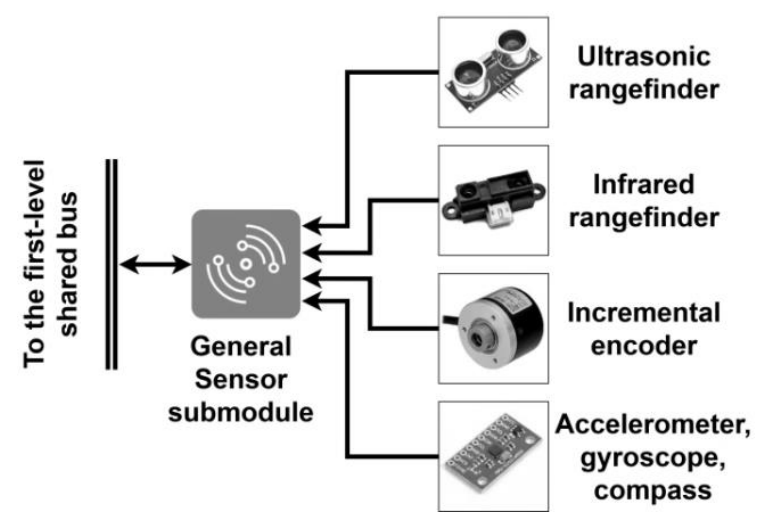

Fig. 4. The connection of various standard sensors to the General Sensor submodule

\section{Laboratory prototypes of submodules and the communication protocol}

While working on the project, prototypes of the Cognition submodule, a DC motor submodule, and a Power module were made. The so-called Base submodules for testing and debugging the communication protocol were also developed and manufactured.

All developed prototypes of submodules have the following main components: the microcontroller STM32 ARMCortex M3 with a frequency of $72 \mathrm{MHz}, 20 \mathrm{~KB}$ of RAM, and $64 \mathrm{~KB}$ of flash memory, a driver for the TJA1050 CAN bus, and a step-down DC-DC voltage Converter.

Cognition submodule: the prototype (in addition to the basic components) includes a Wemos D1 mini controller for wireless communication between the modular robot (transport module) and the user's computer. The USART interface is used for debugging Cognition and other submodules.

DC submodule: the current prototype is capable of controlling motors with a supply voltage of up to $35 \mathrm{~V}$ and a rated current of up to $4 \mathrm{~A}$. Closed-loop control with the shaft's position and/or speed feedback is implemented if the encoder is connected to the submodule. The DC submodule publishes information about the speed and position of the shaft to the bus with a specified frequency (the publication frequency is determined by the cognitive submodule or the user's computer). Figure 5 shows the first version of the prototype of the DC submodule.

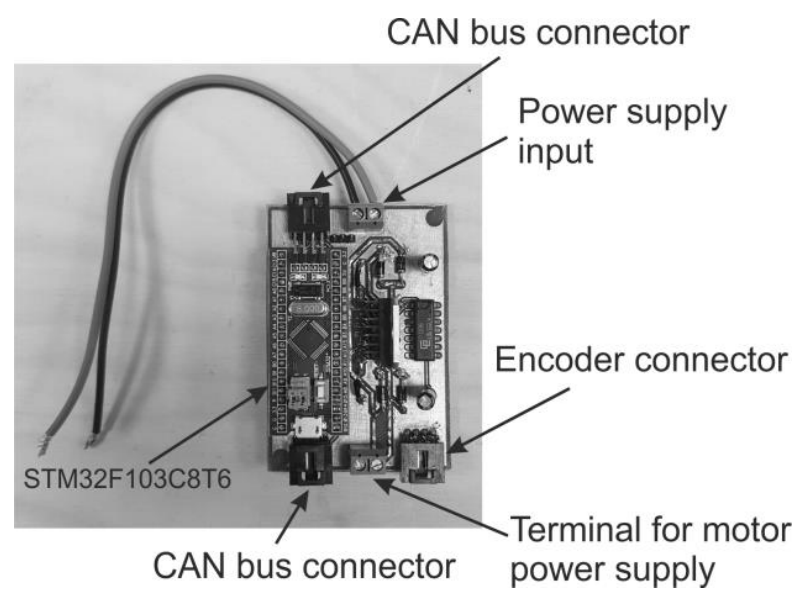

Fig. 5. The first prototype of DC submodule 
Power module: the current prototype module includes lithium-ion batteries, two ATTiny85 microcontrollers, a DCDC voltage Converter, and auxiliary electronic components. The output voltage of the module is in the range of 10-16. $8 \mathrm{~V}$, the current is limited to $5 \mathrm{~A}$ in continuous mode, and $10 \mathrm{~A}$ in peak mode. In current experiments, low-power motors are used, so these characteristics fully meet the requirements. Several Base submodules were manufactured to test the inter-module communication protocol and debug the submodule control algorithms.

The Base submodule (BASE_0) is an electronic device that includes only the basic components inherent to all submodules: a microcontroller, a CAN driver, and a power converter. Also, the device board has sockets for connecting to the bus and auxiliary electrical contacts that duplicate the pins of the microcontroller. Figure 6 shows a photo of four manufactured Base submodules connected via the bus.

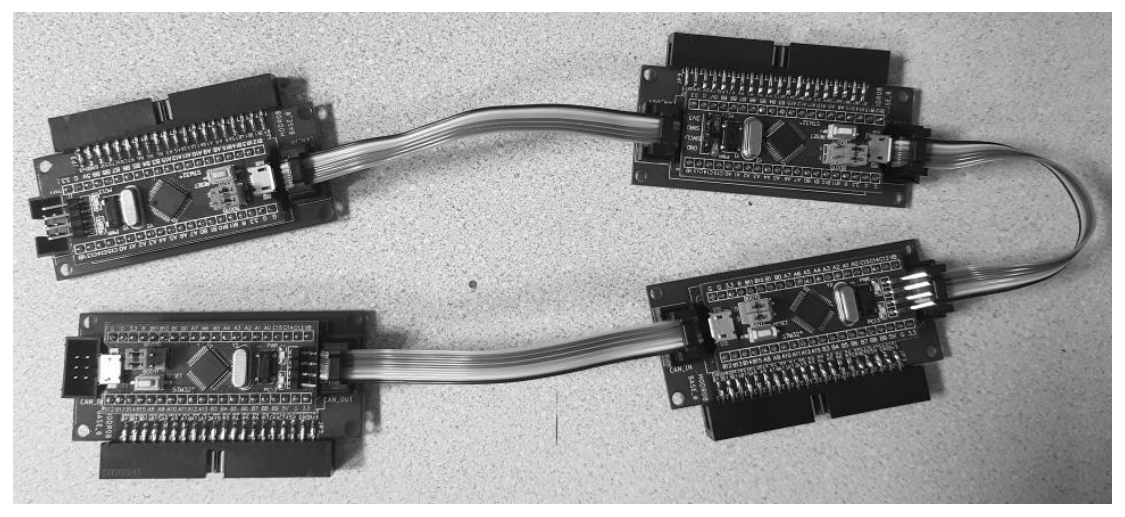

Fig. 6. Base submodules connected to the CAN bus via flat cable

The Base submodule is a universal device that can perform the functions of other submodules when connecting the necessary peripherals and loading the corresponding control program. All submodules are connected to the CAN bus. The choice of this standard is due to its following advantages: consideration of message priority when publishing simultaneously, ease of implementation, high noise immunity, collision detection and error control. In addition, the CAN bus allows any module to become a master through network access arbitration. The disadvantages include a small payload size (only 64 bits, excluding all auxiliary service information) and a small bus bandwidth (up to $1 \mathrm{Mbit} / \mathrm{s}$ ). However, with the hierarchical organization of the control system of a modular mobile robot, while observing the principle of full functionality of modules, the volume of inter-module information interaction is small, which makes it possible to use such a well-established standard. Let's briefly consider the inter-module interaction protocol developed by the authors.

A software node is deployed on each hardware submodule. Each node has its ID (modID) and a collection of variables with their IDs (varID). The protocol is based on the principle of "binding" variables: when a given variable of one module changes, other variables of the modules that subscribed to it will change. To do this, a message containing information about the new value of the specified variable must be published with a non-zero frequency. A software node can receive and publish multicast messages. Each message occupies one extended CAN frame (128 bits). The message contains the following basic information: the module address, the command interpretation bit, the variable value, and the module type specifier. Messages are sent to all bus nodes, but they are processed if the message is intended for the target submodule. The incoming message is interpreted as either "set" or "update". Depending on the message mode, the set-message can be:

- $\quad$ write the value to a receiver variable (by the variable ID),

- $\quad$ set the publication frequency on a bus of a receiver variable (by the variable ID),

- $\quad$ subscribe a receiver variable to a publisher's variable (using two IDs) - variables "binding”.

The update-message works as follows: the receiver variable that was previously subscribed to the publisher variable (by the set-message) gets a new value (updated). The Cognition submodule connects to the top-level computer via a separate communication channel. Currently, this channel is USART. Commands are sent from the computer to the Cognition submodule ("down" in the hierarchy) via the serial port (USB-USART). Such commands can be, for example, setting the target speed of rotation of the motor shaft of the DC submodule. Depending on the task the Cognition submodule can determine which submodules should publish messages and which ones should be accepted. Commands from the computer are similar to installation messages: they have similar information fields. All commands are written in JSON format. This choice is due to the wide popularity of the format and its ease of human perception. Only three types of messages are used:

1. \{"op": "setValue", "modID": Int16, "varID": Int8, "value": Float - set a variable value with identifier varID of a module with identifier modID.

2. \{"op": "setPublication", "modID": Int16, "varID": Int8, "frequency": Int - set the publication frequency (Hz) of a variable with varID of a module with modID $(0-$ no publication $)$. 
3. \{"op": "setSubscription", "modID": Int16, "varID": Int8, "subModID": Int16, "subVarID": Int8\} - set subscription: variable with varID of a module with modID subscribes to a variable with subVarID of a module with subModID that is when changing the subVarID variable, the variable with the varID identifier will be changed if the publication frequency of the variable with the subVarID identifier was set to non-zero before.

Sending up low-frequency messages published on the CAN bus (to the upper-level computing device) is carried out by aggregating data on the Cognition submodule. Aggregated data is also presented in JSON format.

\section{Experiments}

The submodules were tested using the developed protocol. The purpose of the experiment is to investigate the possibility of using the developed protocol for submodules control in real time. Real-time refers to a "problem, system, or application that is concurrent and has timing constraints whereby incoming events must be processed within a given timeframe" [25]. This time period largely depends on the performance of a particular robotic system. In this situation, one should determine the maximum delay that will occur due to the processing of incoming messages by submodules. This delay will determine the actual performance of the system. The scheme of the experiment is presented in the figure 7 .

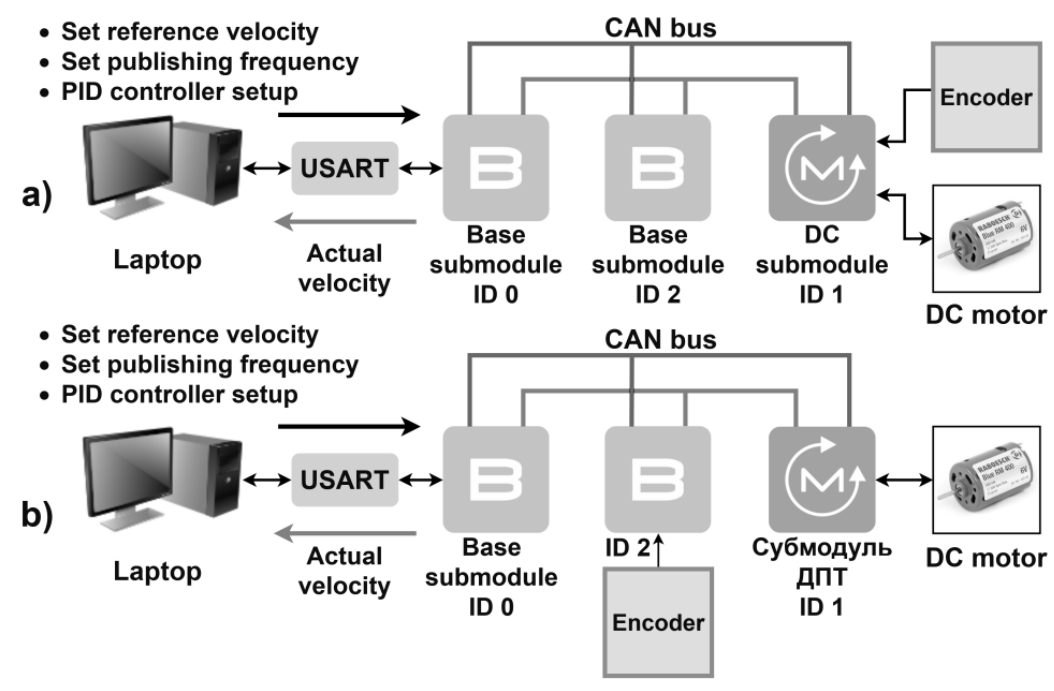

Fig. 7. The scheme of the experiment: a) speed loop of the DC submodule is closed on the DC submodule itself; b) speed loop of the DC submodule is closed via intermediate submodule

The equipment configuration for the experiment is shown in table 1.

\begin{tabular}{|c|c|c|}
\hline Equipment & Quantity & Main parameters \\
\hline $\begin{array}{c}\text { Laptop (upper-level } \\
\text { computing device) }\end{array}$ & 1 & Operating system Manjaro Linux 19.0.0 \\
\hline Base submodule & 3 & See section 4 \\
\hline Motor driver L298N & 1 & Power supply: 5-46 V, max. continuous current: 2 A \\
\hline Integrated chip 7402 & 1 & Four logic gates "negative OR" \\
\hline $\begin{array}{c}\text { Optical incremental encoder } \\
\text { HOA0901-012 }\end{array}$ & 1 & $\begin{array}{c}\text { Resolution to 0.229 mm, operating supply voltage: } \\
\text { 5 V }\end{array}$ \\
\hline Code disc & 1 & Resolution: 30 pulses per revolution. \\
\hline $\begin{array}{c}\text { DC motor Raboesch Blue } \\
\text { RM-410 }\end{array}$ & 1 & $\begin{array}{c}\text { Nominal voltage: 7.2 V, nominal speed: } 7500 \mathrm{rpm}, \\
\text { nominal current: } 1.3 \mathrm{~A}, \text { nominal torque: } 0.022 \mathrm{Nm}\end{array}$ \\
\hline
\end{tabular}

Table 1. The equipment configuration for the experiment

Note: there is no DC submodule in the equipment table, however, it is present in the diagram in Fig. 7. This is due to the fact that the DC submodule in this experiment is implemented on the basis of the Base submodule, as well as the L298N motor driver and the 7402 integrated circuit. The experiment setup. The task is to control the speed of rotation of the DC motor shaft. The experiment was divided into two parts. In the first part (Fig. 7, a), the electric motor and the encoder are connected to the DC submodule (ID 1), i.e. the speed feedback is closed on this submodule. In the second part, the encoder is connected to another submodule with ID 2 (Fig. 7, b), i.e. the speed feedback is closed using this submodule. In both the first and second parts, setting the target shaft rotation speed, as well as setting the publication and subscription frequencies, is performed using a computer connected to the first Base submodule (ID 0) via USART. 
The first part of the experiment. The DC submodule measures the speed of rotation of the motor shaft and writes its value to the variable. The "setPublication" command sent by the computer sets the variable publishing frequency to $100 \mathrm{~Hz}$. The DC submodule actuator must servo control the reference step velocity inputs: 100, 500, 800, 250, -250, $800,-500,-100 \mathrm{rad} / \mathrm{s}$. The desired speed is set by the "setValue" command. The DC submodule only implements a speed loop with a PID controller. Note that the PID controller is not configured for optimal operation. Table 2 shows the parameters of the laboratory setup for the first experiment.

\begin{tabular}{|c|c|}
\hline Parameter & Value \\
\hline CAN bus bandwidth, Mbit/s & 1 \\
\hline USART baud rate, bps & 115200 \\
\hline DC submodule speed loop working frequency, Hz & 100 \\
\hline Publication frequency on a bus of a variable containing speed information, Hz & 100 \\
\hline Proportional coefficient of a PID controller & 3.5 \\
\hline Integral coefficient of a PID controller & 9 \\
\hline Derivative coefficient of a PID controller & 0.2 \\
\hline
\end{tabular}

Table 2. The parameters of the laboratory setup for the first experiment

Note: the measured speed is averaged over 10 points. Figure 8 shows a continuous line which is the angular velocity of the motor shaft. The dashed line shows the desired speed.

The second part of the experiment. As in the first experiment, the DC submodule actuator must servo control the reference-input signals of velocity. Speed measurement is performed in a submodule with ID 2 (to which the encoder is connected); publication is performed at a frequency of $100 \mathrm{~Hz}$. To close the feedback loop, the DC submodule is subscribed to the speed variable of the submodule with connected encoder using the "setSubscription"command. The initial parameters of the laboratory setup for the second experiment are the same as for the first. Figure 8 shows a dashdotted line which is the speed of rotation of the motor shaft obtained during the second experiment.

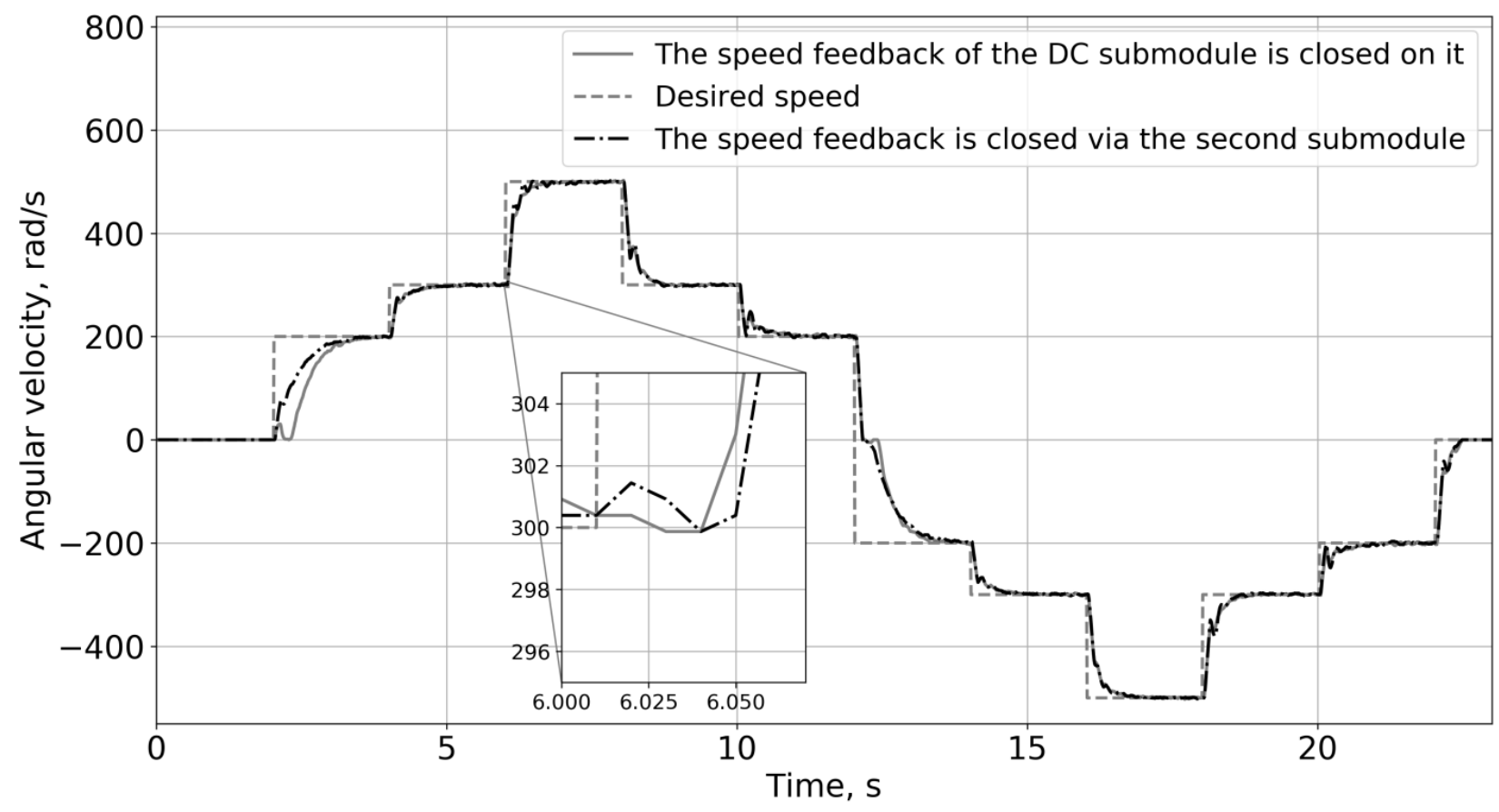

Fig. 8. Comparison of motor speeds in the first and second part of the experiment

The graphs in fig. 8 show that the quality of speed step responses in the second experiment did not change much in comparison with the first experiment, but in comparison with the first experiment, a delay was added to the response delay of the drive to the reference-input. The delay is caused by the presence of a submodule in the feedback loop. The main reasons for the delay are: speed measurement with a period of $10 \mathrm{~ms}$, publication with a period of $10 \mathrm{~ms}$, and processing of incoming messages by the receiver, which occurs with a period of $10 \mathrm{~ms}$. The time spent on sending a message over the bus (about 14-16 microseconds) makes a small contribution to the overall delay duration. Thus, the maximum delay should be about $40-50 \mathrm{~ms}$. Analysis of the graphs showed that the actual delay varies in the range from $4 \mathrm{~ms}$ to $50 \mathrm{~ms}$, which corresponds to the above estimation. At the initial moment of time (with reference-input of $200 \mathrm{rad} / \mathrm{s})$, the reaction delay in the second experiment was even less than in the first (by $0.25 \mathrm{~s}$ ). 
There were also minor oscillations (but within the established error of 5\%) - 2-3 complete oscillations during regulation at speeds of more than $200 \mathrm{rad} / \mathrm{s}$. The experiments were repeated for a sinusoidal reference-input with a frequency of $0.5 \mathrm{~Hz}$ :

$$
\omega_{\text {ref }}=500 \cdot \sin (\pi t)
$$

$\omega_{\text {ref }}-$ reference speed, $\mathrm{rad} / \mathrm{s}$.

All other parameters of the experiment remained the same, except for the parameters of the PID controller, which became equal $K_{P}=6, K_{I}=20$ and $K_{D}=0.3$. Figure 9 shows the motor shaft angular velocities for the first and second part of the experiment respectively for sinusoidal reference-input.

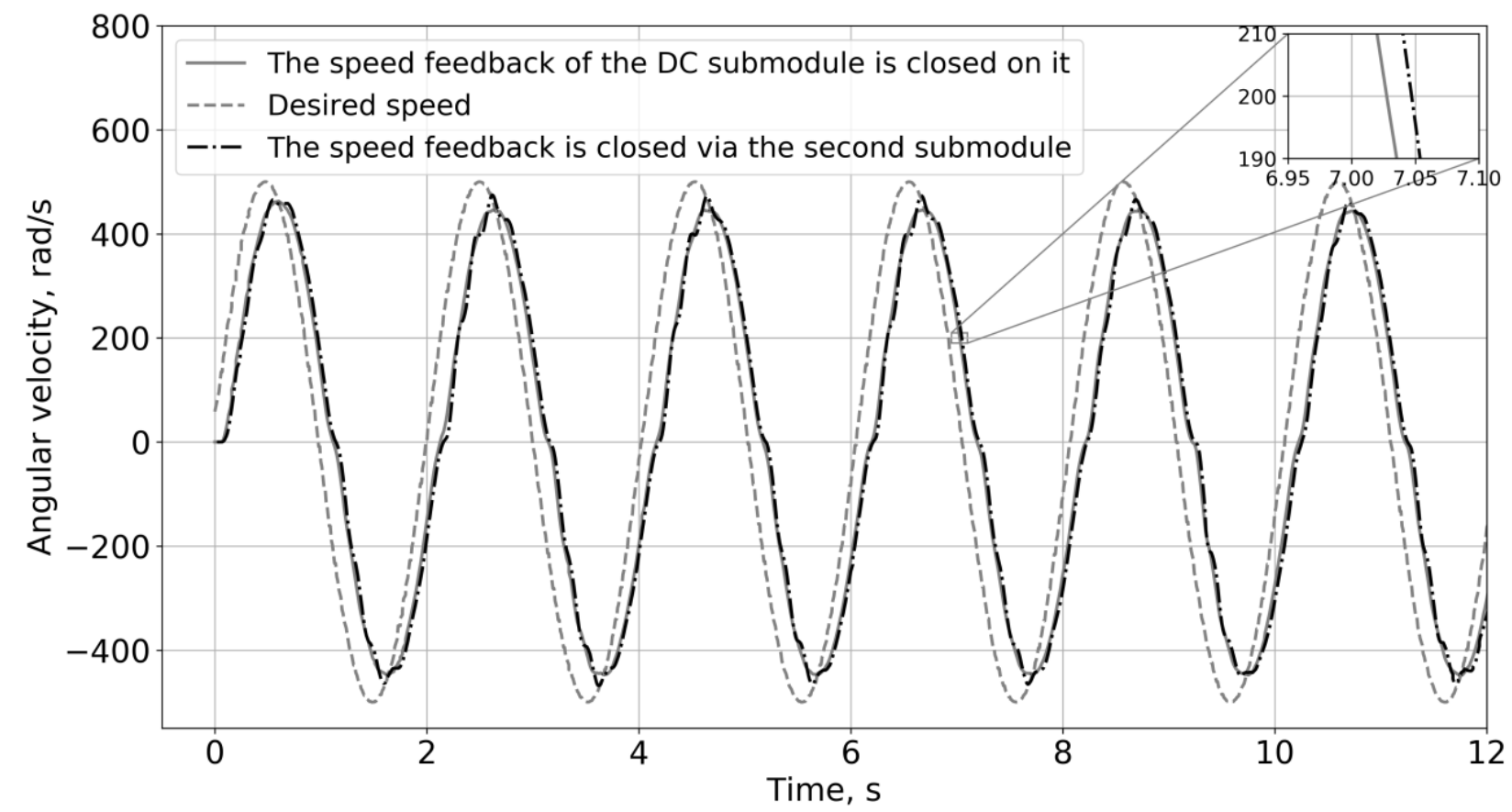

Fig. 9. Comparison of motor speeds in the first and second part of the experiment for sinusoidal reference-input

As in the previous case, the speeds in the first and second experiments differ slightly; however, there is also a delay in the response of the drive. The delay ranges from 13 to $25 \mathrm{~ms}$ (at the initial time). The average delay is $20 \mathrm{~ms}$. Conclusion from the experiment. Analysis of the results showed that the use of the developed inter-module communication protocol allows the real-time control of submodules. Strictly speaking, the experiment is demonstrational, since according to the presented concept, the DC submodule must independently estimate the speed of the electric motor. However, the introduction of an additional submodule in the speed feedback did not disrupt the functioning of the system, although it worsened it within acceptable limits. The drive system remains stable, but an increased delay in response to the reference-input may negatively affect the operation of the drive. This is especially important in high-speed drives of industrial robots, but since the framework we are developing is primarily aimed at mobile robots, this disadvantage is not critical. The speed of most mobile robots working in rooms with people rarely exceeds 1-2 m/s [26], [27], [28]. In this case, the delay in the response of the robot (transport module) to the setting effect can lead to a positional error of the order of $0.8-10 \mathrm{~cm}$, which should not be a big problem if there are remote sensors that publish data to the bus at the same frequency as the submodule with an encoder $(100 \mathrm{~Hz})$.

In this case, the drive response delay can be reduced by increasing the publishing frequency of the speed-variable to the bus. At the same time, the frequency of speed estimation in the submodule with ID 2 should be increased by the same number of times (see fig. 7, b). However, performance improvements can only be achieved if the encoder's code disk has a higher resolution. For the code disk used in this experiment with a resolution of 30 pulses per revolution, increasing the publication frequency to $200 \mathrm{~Hz}$ only worsened the quality of the drive.

It is also worth noting that you can only increase the frequency of publishing variables of a particular submodule up to a certain limit, since the CAN bus will be loaded with messages from other submodules, which can be 5-10 in one transport module. Analysis of the protocol with a large number of submodules actively publishing to the bus is part of further research. 


\section{Conclusion}

This article offers the ModRob framework for developing mobile robots with a modular hierarchical architecture. The main differences between this framework and the ones listed in the survey include:

- hierarchical topology that allows to distribute tasks between modules both vertically and horizontally,

- use of full-featured modules at all levels of the hierarchy: from low-level drive control to high-level mobile platform control.

It is shown that the second level of the hierarchy can be represented by six main full-featured modules, each of which should include several standard submodules, which should ensure ease of integration of third-party devices. The structure of the transport module, consisting of three main standard unified submodules: Cognition, Actuator and General Sensor submodule, is developed. The existence of such a unified sub-modules facilitates the assembly of the various transport platforms. Prototypes of submodules for the first level of the hierarchy were made: the Cognition submodule, the DC submodule, and the Power module. For testing were also designed and manufactured the Base submodules, allowing to debug the operation of a protocol. Experimental studies performed on manufactured submodules have shown that connecting submodules with unique identifiers to the bus allows the system to start working in plug-and-play mode, i.e. without additional setup.

A protocol for inter-module communication has been developed that uses a minimum set of standard messages, which made it possible to use the CAN bus to ensure the functioning of submodules in real time - there is a guarantee that the delay introduced by a separate submodule when transmitting messages does not exceed 50 ms. However, it should be noted that this is true under the conditions mentioned above (set of equipment and submodules).

The proposed approach and the results of experimental research have shown that the division of a full-featured transport module into submodules, based on the hierarchical principle of building a multi-level control system for a modular mobile robot, allows to increase the performance of the entire system by distributing the computational load between modules and submodules when using embedded systems in each module and submodule (relatively cheap microprocessors or single-board computers of low performance).

Future work. A significant part of further work will consist in investigating a system with a large number of submodules: determining the dependence of message reception delays and their transmission speed versus the number of subscribers, the magnitude of jitters in packet transmission delays, etc. This is necessary because a single transport platform can have up to 10 submodules that actively publish data at a frequency of $100 \mathrm{~Hz}$ or more.

An important task is to make other submodules (for example, a General Sensor) and debug them, which will allow to further implement the prototype of the transport module, which includes all the units mentioned above.

\section{Acknowledgments}

The Russian Foundation for Basic Research supports this research: Grant 19-07-00892a.

\section{References}

[1] Phidgets Inc. - Products for USB Sensing and Control, URL: https://www.phidgets.com/ (accessed: 26.02.20).

[2] HEBI Robotics, URL: https://www.hebirobotics.com (accessed: 26.02.20).

[3] Andreev, V[ictor]; Kim, V[alerii] \& Pryanichnikov, V[alentin] (2019). A Hierarchical Modular Architecture for Reconfigurable Mobile Robots, Proceedings of the 30th DAAAM International Symposium, pp.1152-1159, B. Katalinic (Ed.), Published by DAAAM International, ISBN 978-3-902734-22-8, ISSN 1726-9679, Vienna, Austria. DOI: 10.2507/30th.daaam.proceedings.162.

[4] Iñigo-Blasco P., Diaz-del Rio F., Romero-Ternero M., Cagigas-Muñiz D. \& Vicente-Diaz S. (2012). Robotics software frameworks for multiagent robotic systems development, Robotics and Autonomous Systems, Vol. 60, No. 6, 2012, pp. 803-821, ISSN: 0921-8890.

[5] Herbrechtsmeier S., Korthals T., Schopping T. \& Ruckert U. AMiRo: a modular \& customizable open-source mini robot platform // 20th International Conference on System Theory, Control and Computing (ICSTCC), Sinaia. 2016. pp. 687-692.

[6] Kalouche S., Rollinson D. \& Choset H. Modularity for maximum mobility and manipulation: control of a reconfigurable legged robot with series-elastic actuators // IEEE International Symposium on Safety, Security, and Rescue Robotics (SSRR). - West Lafayette, IN, USA, 18-20 Oct. 2015. - pp. 1-8, IEEE, New York.

[7] Virk G. (2003). CLAWAR Modularity for Robotic Systems, The International Journal of Robotics Research, Vol. 22, No. 3-4, March-April 2003, pp. 265-277, ISSN: 02783649.

[8] Steinbauer G., Wotawa F. \& Fraser G. (2004). A modular architecture for a multi-purpose mobile robot, Innovations in Applied Artificial Intelligence. IEA/AIE 2004. Lecture Notes in Computer Science, ISBN 978-3540-24677-0, pp. 1007-1015, Springer, Berlin, Heidelberg, DOI: 10.1007/978-3-540-24677-0_103.

[9] Taira T., Kamata N. \& Yamasaki N. (2005). Design and implementation of reconfigurable modular humanoid robot architecture, IEEE/RSJ International Conference on Intelligent Robots and Systems 2005, 2-6 Aug. 2005, Edmonton, Alta., Canada, ISSN 2153-0858, pp. 1071-1076, IEEE, New York, DOI: 10.1109/IROS.2005.1545122. 
[10] Ahn H., Beak Y., Sa I., Kang W., Na J. and Choi J. (2008). Design of reconfigurable heterogeneous modular architecture for service robots, IEEE/RSJ International Conference on Intelligent Robots and Systems 2008, 22-26 Sept. 2008, Nice, France, ISSN 2153-0858, ISBN 978-1-4244-2057-5, pp. 1313-1318, IEEE, New York, DOI: 10.1109/IROS.2008.4650706.

[11] Hild M., Siedel T., Benckendorff C., Thiele C. \& Spranger M. (2012). Myon, a New Humanoid, In: Language Grounding in Robots, Steels L., Hild M. (eds), pp. 25-44, Springer, ISBN 978-1-4614-3063-6, Boston, MA.

[12] Roh S., Yang K., Park J., Moon H., Kim H.-S., Lee H. \& Choi H. (2009). A modularized personal robot DRP I: design and implementation, IEEE Transactions on Robotics, Vol. 25, No. 2, 2009, pp. 414-425, ISSN 1552-3098, DOI: $10.1109 /$ TRO.2009.2014499.

[13] Magnenat S., Rétornaz P., Bonani M., Longchamp V. \& Mondada F. (2010). ASEBA: a modular architecture for event based control of complex robots, IEEE/ASME Transactions on Mechatronics, Vol. 16, No. 2, 2010 , pp. 321 329, ISSN 1083-4435, DOI: 10.1109/TMECH.2010.2042722.

[14] Bonarini A., Matteucci M., Migliavacca M. \& Rizzi D. (2014). R2P: An open source hardware and software modular approach to robot prototyping, Robotics and Autonomous Systems, Vol. 62, Issue 7, 2014, pp.1073-1084, ISSN 0921-8890, DOI: 10.1016/j.robot.2013.08.009.

[15] Losada, D.P., Fernández J.L.; Paz E. \& Rafael S. (2017). Distributed and modular CAN-based architecture for hardware control and sensor data integration, Sensors, 17(5). 2017, pp.1013-1030, ISSN 1424-8220, DOI: $10.3390 / \mathrm{s} 17051013$.

[16] Shmakov O., Korolev D., Popov D., Kitaev N. \& Korotkov A. (2017). Modular Mobile Robotic Kit for Prototyping and Debugging of Control Algorithms, Proceedings of the 28th DAAAM International Symposium, pp.0950-0956.

[17] Mayoral V., Hernandez A., Kojcev R., Muguruza I. Zamalloa I. Bilbao A. \& Usategi L. (2017). The shift in the robotics paradigm - the Hardware Robot Operating System (H-ROS); an infrastructure to create interoperable robot components, NASA/ESA Conference on Adaptive Hardware and Systems (AHS) 2017, Pasadena, CA, USA, 24-27 July 2017, ISSN 2471-769X, ISBN 978-1-5386-3439-4, pp.229-236, IEEE, New York, DOI: 10.1109/AHS.2017.8046383.

[18] Jahn U. Wolff C. \& Schulz P. (2019). Concepts of a modular system architecture for distributed robotic systems, Journal of Computers, Vol. 8, Issue 1, 2019, 16 pages, ISSN 2073-431X, DOI: 10.3390/computers8010025.

[19] Andreev V. Control System Mobile Robots with Modular Architecture as a Multi-Agent System with a Hierarchical Topology, Proceedings of the 30th DAAAM International Symposium, B. Katalinic (Ed.), Published by DAAAM International, Vienna, Austria. 2019. pp.0010-0019.

[20] Andreev, V., Kim V. \& Pletenev P. The Principle of Full Functionality - the Basis for Rapid Reconfiguration in Heterogeneous Modular Mobile Robots, Proceedings of the 28th DAAAM International Symposium, B. Katalinic (Ed.), Published by DAAAM International, Vienna, Austria. 2017. pp.0023-0028

[21] Andreev V. \& Kim V. Control system and design of the motion module of a heterogeneous modular mobile robot. 27th International DAAAM Symposium on Intelligent Manufacturing and Automation 2016, Proceedings of a meeting held 26-29 October 2016. Mostar, Bosnia and Herzegovina, B. Katalinic (Ed.), Curran Associates, Inc. 2017. pp. 0586-0594.

[22] Yurevich Ye.I. Osnovy robototekhniki: Uchebnik dlya vtuzov [Fundamentals of Robotics: Textbook for Technical Universities] - Leningrad: Izd-vo Mashino-stroyeniye, Leningr. otdeleniye, 1985. - 271 p. (In Russ).

[23] Siciliano B. Sciavicco L. Robotics Modelling, Planning and Control / B. Siciliano [et al.]. - Springer-Verlag London Limited, 2009. - 632 p.

[24] Siegwart, R. Introduction to Autonomous Mobile Robots / R. Siegwart, I. Nourbakhsh, D. Scaramuzza. Cambridge: The MIT Press, 2004. - $472 \mathrm{p}$.

[25] International Standard ISO/IEC/IEEE 24765:2017 Systems and software engineering - Vocabulary, second edition 2017-09.

[26] Service robot Care-O-bot 4, URL: https://www.care-o-bot.de/de/care-o-bot-4/technical-data.html/ (accessed: 26.02.20).

[27] Kiva Systems: Three Engineers, Hundreds of Robots, One Warehouse, URL: https://spectrum.ieee.org/robotics/robotics-software/three-engineers-hundreds-of-robots-one-warehouse (accessed: 26.02.20).

[28] Service humanoid robot Rollin' Justin, URL: https://www.dlr.de/rm/en/desktopdefault.aspx/tabid11427/\#gallery/29202 (accessed: 26.02.20). 